\title{
Construction and Practice the Wikipedia System on Educational Administration Management in Colleges and Universities
}

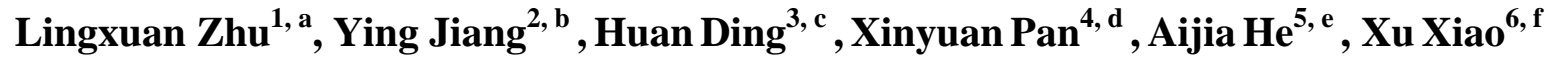 \\ School of Management, Beijing Normal University, Zhuhai 519087, China. \\ azhulingxuan@bnuz.edu.cn, bjpz6311whu@bnuz.edu.cn,'dinghuan@bnuz.edu.cn \\ d997175472@qq.com,eaijia.he@qq.com,,xuxiao_chn@yeah.net
}

Keywords: Wikipedia; Educational Administration; Teachers’ Manual.

\begin{abstract}
In order to adapt to the education management status that the numbers of changes in the management system and documents increased significantly, based on the purpose of innovation and entrepreneurship education, this paper puts forward the new requirements of educational administration in universities and using collaboration, sharing, participation advocated by Wikipedia. Through practicing two phases system projects in "Teacher's Manual of Management academic, Beijing Normal University, Zhuhai campus(BNUZ)”, finally reduced the number of educational administration documents to $62 \%$ and rules to $59.92 \%$. The result shows that Wikipedia is suitable for knowledge management, integration and evolution under the people collaboration.
\end{abstract}

\section{Introduction}

With the advancement of higher education reform, various of new system documents are introduced. It is more complex and difficult to manage the educational knowledge due to large number of terms, documents and requirements. In Management academic of BNUZ, there have 170 even 200 courses were opened per semester, average 50 students in a course and each student needs to complete two presentations at least, which involves more than 40 teachers, the data that relevant teaching and system documents reach to "million". Besides, some different documents contain a lot of similar contents, it is usual that teacher needs to find multiple documents in order to ensure information integrity when he uncertain, but information may also be missed in searching. It is obvious that traditional methods in managing educational knowledge are detrimental to development. Therefore, the innovation of educational management in universities is necessary. Knowledge management mainly refers to building a humanistic and technical knowledge system in a organization, through acquiring, creating, sharing, integrating, recording, accessing, updating and other processes between information and knowledge to achieve knowledge innovation and give back to the knowledge system. The Wikipedia has these characteristics that meets the current needs of knowledge management in universities. It has been used in the library, government, business and other knowledge management. In this context, this paper made Wikipedia into educational knowledge management and achieved a teacher's manual, become the educational administration simple and accurate, which meets the current trend of the big data age.

\section{The Current Research Situation Analyzing}

Zheng Yi, who proposed using knowledge management can increase the efficiency and adaptability of logistics sector. Knowledge management helps to improve the overall efficiency of modern logistics: a company has new technologies cannot be popularized and promoted in other companies. Knowledge management devotes to allocating the knowledge resources, classification and dissemination, to facilitating people to explore the knowledge already owned, through using the available knowledge to the new problems, so that knowledge resources can be fully reused, further to improve logistics' overall efficiency [1]. Knowledge management is by using sharing, making the hidden knowledge apparent to learn and progress. Traditional educational administration has 
defects: educational administration work is hierarchical, lots of useful knowledge uncontrolled. Increasing participatory can manage information and improve horizontal macro-control when knowledge management was embedded into traditional educational administration management [2]. With the large data age, the pure knowledge management is unable to satisfy the new needs, it begins to have new innovative researches boost the information management into a new stage by combining Wikipedia platform with knowledge management methods. Alden put forward that using Wikipedia to manage knowledge has "dynamic" attribute, it is more simply to search, view and modify information [3].

Qian Yan found that the Wikipedia platform is significant for knowledge management updating. Knowledge management based on Wikipedia truly realized the knowledge sharing and made the quality of network information greatly improved, also helped to build the team knowledge management platform, and further completed the personal knowledge management system [4]. It is a new breakthrough that the realization of knowledge sharing becomes the hidden knowledge into dominant, while was shared and updated through the platform.

Through a large number of theoretical research, people began to contact the practical research of Wikipedia in all areas: Liu Jing taken use of Wikipedia to build a database linked to navigation page and realized library's advisory services. Long-term practice study presents the Wikipedia platform can complete the model that information acquisition and sharing service, further to achieve one-to-many and time-limited service model, so that it can reduce the library's pressure and assure users to get more comprehensive services [5]. A knowledge management system model based on semantic Wikipedia is constructed by Xiaoyun Zhang, she analyzed the value chain of the system, by using MediaWiki and SMW to realize knowledge management system's prototype and made the knowledge visualization [6]. Hepp M proposed the Wikipedia platform's collaborative editing function can increase the speed of knowledge updating and promote knowledge self-organization. Researchers found the MediaWiki's outstanding convenience when they recorded catalogue data through MediaWiki [7]. Chu S K W discovered Mediawiki is simple, convenient and easy to operate instead of TWiki by comparing experimental results [8].

\section{Current Situations and Problems of Educational Administration Files in BNUZ.}

There have following questions in educational administration system files(system files) of BNUZ: (1)The code of the system files lacks of standardization. It has not been numbered when a new system file introduced. (2)The old and the new system files coexisting. Some expired files have not been promptly abolished, with the accumulation of time, the system files piled up and formed a jumble situation. (3)The contents of the system files scattered. Teacher needs to look for multiple documents to ensure information integrity, but some information is usually missed. (4)It is difficult that some files few used to be found out. (5)The operation of the files is not clear. (6)Parts of the system file updating is not marked with the date. Some of the new policy documents introduced as the supplementary documents and contradict with the old. (7)Some terms are hidden in tabulations instead of existing in a corresponding file.

In summary, it is necessary to sort out knowledge and implement the knowledge management in universities' educational administration.

Building the "Teacher’s Manual of Management Academic in BNUZ”.

This article selected WikiDot and MediaWiki to build two phases teacher's manual.

1.Principles of Construction

(1)The purpose of “Teacher's Manual” is convenient to implement teaching, it arounds the knowledge associated with teaching and teachers.(2)The "Teacher's Manual" was established only for the management academic teachers in BNUZ.(3)The establishment of "Teacher's Manual" improved constantly with data systems and other files updating.(4)“Teacher's Manual” includes two parts of documents in BNUZ management academic.

2. Constructed Phase I Project Based on WikiDot Teacher's Manual. 
It mainly was completed by ten teachers from BNUZ management academic. Two teachers moved the system files to the page"List of Teaching Documents". One teacher controlled the progress and coordinated others. One teacher managed educational administration and divided the teaching files into 31 initial WikiDot pages. Distributed the system documents equally to the other seven teachers. They excerpted the terms associated with the initial WikiDot page contents, then put these statements into the initial WikiDot pages, while marking the source of sub-statement(As is shown in Figure 1). When clauses were extracted, they used the following syntax in the original file to mark them: -delete the content-, it represents the statement had been removed, it brought the convenience for replacing files updated in future.The last teacher was responsible for collating the conflicting statement in original WikiDot page, and finally got the integrity WikiDot page.

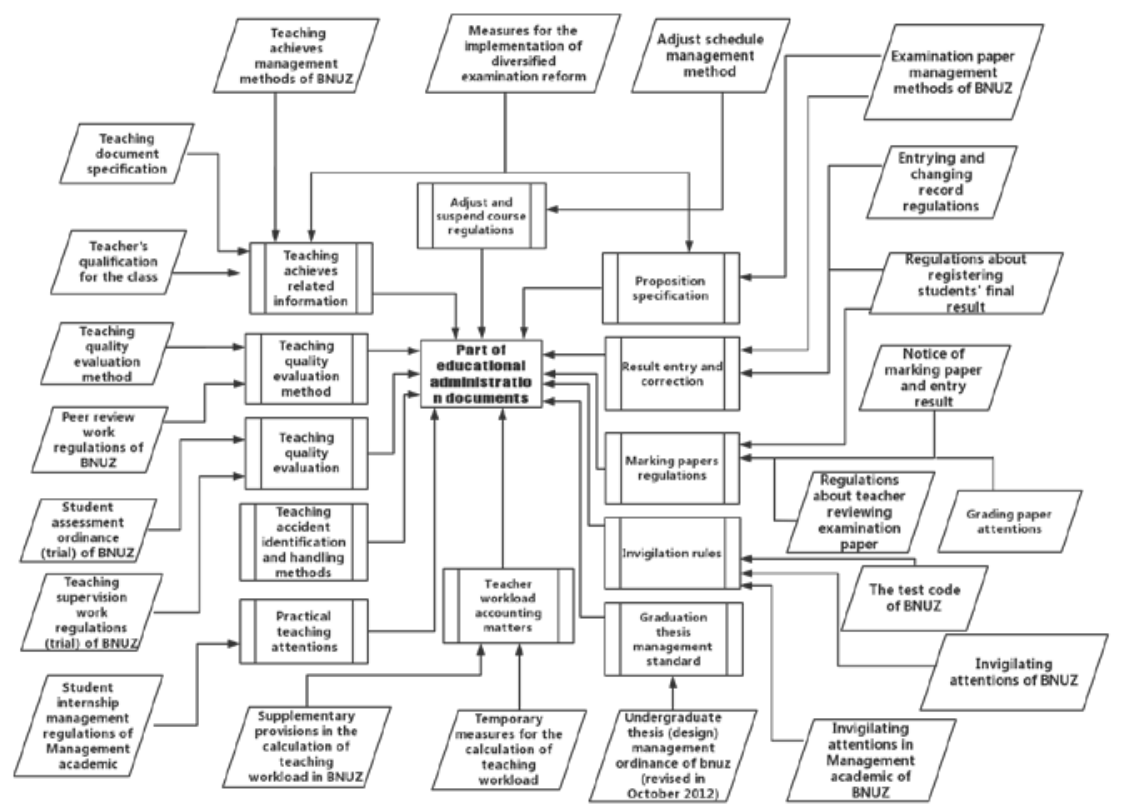

FIG.1 Educational administration documents knowledge diagram

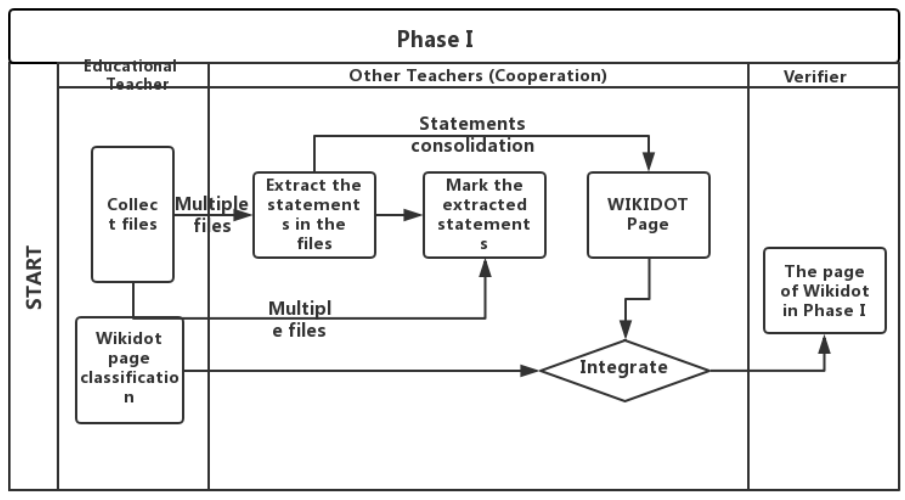

FIG.2 Flow chart of phase I

In Figure 2, the WikiDot page can be acquired from the same original file and different original files so that the chaotic information becomes unified and clearly.The concise grammar, easy to operate and learn are the advantages of WikiDot. If people accidentally delete the required content during the editing process, they can view the WikiDot edit history to get the original information.In the excerpt process, WikiDot highlights its sharing, people can simultaneously modify the published WikiDot page, which makes the work's efficiency greatly improved.

In the process, a total of 135 pages were edited 1627 times. In Table 1, jpz6311whu edited most times(465 times). The efficient work also benefited from WikiDot's collaborative editing, reducing the teacher's pressure in the first phase of the project. 
Table 1. The number of Wikidot edited by the first phase of the project editors

\begin{tabular}{|c|c|c|c|c|}
\hline $\begin{array}{l}\text { Wikidot } \\
\text { account }\end{array}$ & $\begin{array}{l}\text { Edit } \\
\text { times }\end{array}$ & $\begin{array}{l}\text { The page } \\
\text { was edited } \\
\text { most }\end{array}$ & $\begin{array}{l}\text { Edit the maximum } \\
\text { number of pagess } \\
\text { that edited most }\end{array}$ & $\begin{array}{l}\text { The number } \\
\text { pages were } \\
\text { ediled }\end{array}$ \\
\hline pengweio203 & 81 & $\begin{array}{l}\text { Educational administration } \\
\text { work items }\end{array}$ & 38 & 21 \\
\hline changlijun & 27 & $\begin{array}{l}\text { Specialized course material } \\
\text { matters }\end{array}$ & 6 & 12 \\
\hline ptacchl & 82 & $\begin{array}{l}\text { Attentions about adjusting } \\
\text { and suspending course }\end{array}$ & 40 & 14 \\
\hline PANHAO050 & 230 & $\begin{array}{l}\text { Internship and practice } \\
\text { manual for graduates }\end{array}$ & 60 & 11 \\
\hline jingyatou 1110 & 72 & Directory of teacher's manual & 11 & 15 \\
\hline 961003 shaomaorong & 284 & Proposition specification & 36 & 45 \\
\hline wuaingyuan & 83 & Daily teaching monitoring & 18 & 17 \\
\hline xinzhao & 98 & $\begin{array}{l}\text { Educational administration } \\
\text { work items }\end{array}$ & 23 & 15 \\
\hline jpz6311whu & 465 & Teacher's manual & 56 & 63 \\
\hline dxcui & 113 & $\begin{array}{l}\text { Educational administration } \\
\text { work kitems }\end{array}$ & 15 & 64 \\
\hline
\end{tabular}

3. The Second Phase of “Teacher's Manual” Based on MediaWiki.

WikiDot is hosted on a foreign server and needs to access it. Through trial running the "Teacher's Manual" found that due to the existence of firewalls, domain name resolution and other issues, its has an unstable operation issue at home. Entirely depending on the "Teacher's Manual" WikiDot will cause unnecessary troubles. So we used MediaWiki Wikipedia system to further completing the "Teacher's Manual" in the second phase. MediaWiki is completely built on one server of Management academic, there has no instability in accessing, makes "Teacher's Manual"more secure. In addition, researchers further improved the content of "Teacher's Manual”,it was established by three students under a teacher's leadership.

(1)To build and configure the MediaWiki system: installing MediaWiki is relatively simple, but “Teacher's Manual” requires to reference the internal picture and support file download. It not only needs to add the statements such as "wgUploadDirectory", "\$wgFileBackends" and "\$wgLocalFileRepo" in the file of "locasettings.php", but also need to refer to the "defaultsettings" in the "includes" folder to make the appropriate changes on above statements, to ensure the folder that MediaWiki "Teacher's Manual” saves the files has the same path [9]. When the errors or a number of consecutive distortion errors (usually more than 9 errors reported)happen, it also need to change the related statements path, such as "\$wgTmpdDirectory", "\$wgImageMagickTempDir" and etc, and to verify whether it corresponds to the save path. Beyond that, the most important is to open the permission of image folder 777 [10].

(2)The Construction Process of MediaWiki “ Teacher's Manual”: Firstly, moving the all educational files in "Teacher's Documents list” to MediaWiki. Then, extracting the latest system files from dean's homepage. Migrating these files into the "Teaching Documents List” page. The steps are shown in Figure 3 below.

Table 2. Comparison of engineering features between phase I and phase II

\begin{tabular}{|c|c|c|}
\hline Time limit for a project & Phase I of the project & Phase II of the project \\
\hline Wikipedia system & Wikidot & Mediawiki column \\
\hline System architecture & Abroad, remote & Domestic, local \\
\hline System maintenance cost & No & Higher \\
\hline Stability of operation & Unstable & Stable \\
\hline Constructors & Teacher priority & Student priority \\
\hline Construction cycle & Short & Long \\
\hline Content improvement & imperfect & perfect \\
\hline
\end{tabular}




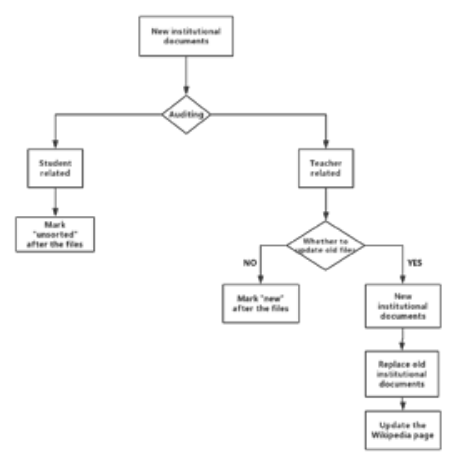

FIG.3 The phase II institutional documents update procedure in project

The second phase of the project system file update will appear the following circumstances:

(1)The system file is only relevant to the student, marking after it if needn't combed. (2)The system file is related to teacher and belongs to the new system files and mark it later:(new). (3)The system file related to the teacher and the old existed before belongs updated content. After confirming and replacing the old system file with the new and mark it at the end(update). Finally checking if the updated clause is marked and needs to find the documentation concluding the clause in the MediaWiki “Teacher's Manual”page and update it.

On the above, using "Templete" to beautify the interface and category documents. Templete is a special kind of module in the Wikipedia system that allows editor quote after editing which can quickly replace and modify plenty of articles [11]. The edited Templete can be directly quoted on pages. One hand, it compressed the number of home codes, speeded up the web page. The other hand avoided looking for a needle in a haystack for modifying a few lines of codes. Templete can more intuitive to allow users to add files and save time. The classification made manual's interface become simple, orderly and beautiful, also convenient for the maintenance in the later.

4. Comparing the Project Between Phase I, and Phase II

In Table 2. The first phase is a preliminary document. The main purpose is to create it and the content is incomplete. While the second phase improved, updated and maintained based on phase I.

\section{Application Test and Practical Effect}
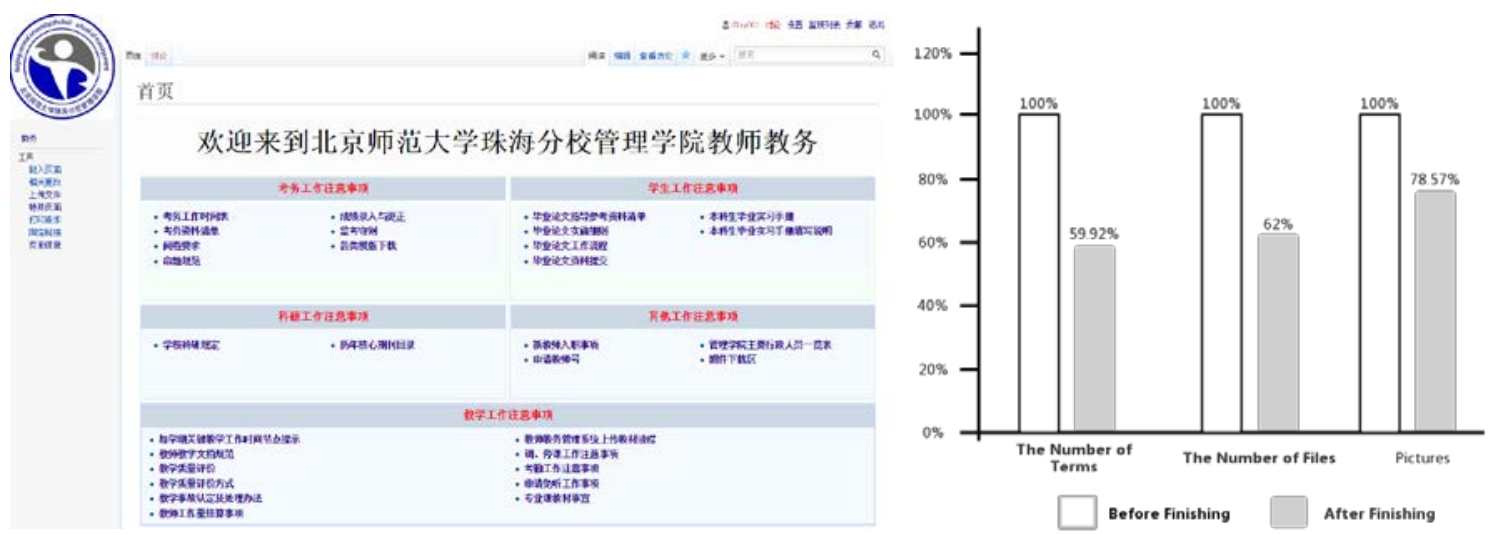

FIG.4 The Home Page of Teacher's Manual based on WikiMedia.
FIG.5 The result in comparing the number of documents in the educational administration system

The home page of MediaWiki “Teacher's Manual” (http://som.bnuz.edu.cn/wiki)as is shown in Figure 4. A total of 302 Wikipedia pages were created, 95 system files were uploaded, 1315 pages were edited, where the average editing number of per page is 4.35. In Figure 6, after knowledge management, files reduced from 50 to 31 copies, articles from 559 to 335, pictures from 70 to 55. 


\section{Summary}

In summary, the four most significant advantages of Wikipedia are:1)Co-edit. The traditional file modification method can only achieve a single individual changes, but Wikipedia can support multi-person cooperation at the same time to modify the page, not only the work efficiency was improved, but also changed the one-to-one information transmission phenomenon. (2) Tracking records and can be restored. Wikipedia can save the edited records, can track the updating information, to ensure accuracy and security, people can modify the error and find the original record to restore the information if uncertain. (3)Easy to operate. Wikipedia grammar is so simple that everyone can use it. (4)High expanding. Wikipedia can add plug-in on the original system for functional expansion, such as multilingual translation, visual editor and video player. These advantages are unattainable by traditional and other ways in teaching knowledge management, which also adapts to the big data age. Thus, Wikipedia is one of the most effective way to realize the knowledge management of educational affairs in universities.

\section{References}

[1] YI Zheng, Jing Wang, Haoyu Wang.Using Knowledge Management to Improve Modern Logistics Efficiency[J]. Marketing modernization, 2012(4):6-7.

[2] Juan Wang, Changshen Hu. Knowledge Management Mode is Integrated into the Strategy of Etching Management in Colleges and Universities[J].Economic Research Guide,2009(35);201-202.

[3] Alden,David D, Wiki Knowledge Management System Documentation[J]. 2010.

[4] Yan Qian.Research on Knowledge Management Application based on Wiki[J].Private Science and Technology,2010(11):194-194.

[5] Jing Liu.Research on Virtual Reference Service System of the University Library based on Wiki[D].Liaoning Normal University,2012.

[6] Xiaoyun Zhang.Research on Knowledge Management System based on Semantic Wiki[J].Lantai World,2015(26):142-143

[7] Hepp M, Siorpaes K, Bachlechner D, harvesting Wiki Consensus: Using Wikipedia Entries as Vocabulary for Knowledge Management[J]. Internet Computing IEEE, 2007, 11(5):54-65.

[8] Chu S K W, Siu F, Liang M, et al. Users' experiences and perceptions on using two wiki platforms for collaborative learning and knowledge management[J]. Online Information Review,2013,37(2):304-325.

[9] Shiping Liu. Establishment of Subject Information Portal Platform based on Mediawiki[J]. Journal of Hunan First Normal University, 2011,02:137-140.

[10] Popescu E, Maria C, Udristoiu A L, Fostering Collaborative Learning with Wikis: Extending MediaWiki with Educational Features[M]// Advances in Web-Based Learning- ICWL 2014, Springer International Publishing, 2014:22-31.

[11] Ying Huang, Zuhua Jiang, Yongwen Huang.Methods of reviewing project experience knowledge recommended by experts[J]. Journal of Shanghai Jiao Tong University, 2015, 49(12):1833-1841. 